Sección dos: Textos

Educación y Exclusión social

\title{
El reflejo de la realidad actual de nuestros menores ante el divorcio. ${ }^{1}$
}

The reflection of reality from our kids and divorce.

\author{
Liliana Núñez Amador \\ María Luisa Vallejo Torres \\ Psicoclinic Sociedad \\ Cooperativa Andaluza \\ gerencia@psicoclinicjerez.com
}

\section{Resumen}

¿Tienen los menores derechos propios y reales, en un periodo de ruptura sentimental de sus progenitores/as? ¿Prevalecen los derechos de los padres sobre los derechos de los/las hijos/as? ¿Cuáles son nuestras responsabilidades con los menores? ¿La responsabilidad como padres o madres acaba cuando se rompe una relación de pareja? ¿Los padres siempre tienen derecho a ver a los/las menores tras una ruptura, independientemente de las circunstancias en las que se produjo la misma?

De un tiempo a esta parte, estas son algunas de las preguntas que, tanto a profesionales como legos, inmersos de una manera u otra en litigios de familia, pueden suscitar interés. Como profesionales de la psicología forense interesadas en esta temática, y atendiendo al elevado número de caso que nos llegan a evaluación, derivados de esta situación, consideramos de especial importancia atender este tipo de cuestiones, así como el abordaje de posibles entornos de riesgo, desde una perspectiva psicoeducativa.

Palabras clave: Divorcio, separación, menores, protección, psicoeducación.

\section{Abstrac}

Do our kids have their own real rights during their parents `sentimental brakage? Are parents 'rights above their kids` rights? What our responsabilities with young sters? Does the

${ }^{1}$ Recibido: 10/06/2018 Evaluado: 11/06/2018 Aceptado: 25/06/2018 
responsability as parents end when a coupleis over? Do parents have always the right to see their kids after a breakage, independently from circunstances?

For some time now these are some of the questions that both professionals and non professionals, involved any how with family disputes, may show interest. As forensic psychologist professionals interested in this subject and following so many cases that arrive for evaluation from this situation, we consider of suchan importance to attend this kind of questions, as to aproveche posible risky environments, from a psychological educational perspective.

Keywords: Divorce, separation, kids, safety, psycoeducation.

\section{Introducción}

Las primeras experiencias vitales de un ser humano, marcan, en un sentido u otro, su trayectoria como adultos. Es por ello que, las madres, padres o todas aquellas personas encargadas de la crianza de los/as menores, poseen una responsabilidad primordial en este aspecto.

El punto de partida del presente escrito, estriba en la primera cuestión planteada. Actualmente, los/as menores gozan de especial protección, pero no siempre fue así. Hasta fechas recientes, los/as niños/as podían ser objetos impunemente de castigos físicos, limitaciones serias de su libertad y prácticas educativas dañinas amparadas en costumbres culturales. Hoy en día, estas situaciones siguen produciéndose en algunos países y culturas; no obstante, en países "desarrollados" como el nuestro, los/as menores son considerados como sujetos de plenos derechos (Convención del 20 de noviembre de 1989, Asamblea General de Naciones Unidas. Convención de los Derechos del Niño, convirtiéndose en la Ley del 02 de septiembre de 1990). Derivado de este consenso, los/as niños/as tienen una especial protección y sus derechos prevalecen sobre cualquier otra persona o situación, no predominando los de sus progenitores, sobre el de los hijos/as.

Es un hecho al alcance de todos, ser conscientes y considerar el creciente número de separaciones y divorcios desde las últimas modificaciones del Código Civil. Estas reformas trajeron consigo una serie de reformas y cambios de perspectivas, que siguen en constante evolución, derivadas de las demandas de la sociedad. Son muchos los profesionales inmersos en esta temática (jueces/as, letrados/as, mediadores/as, psicólogos/as, estos últimos en todas sus modalidades, tales como peritos, expertos, sanitarios, etc.), abogando por un abordaje multidisciplinar, especialmente en el tema que nos ocupa, el papel protagonista los niños y las niñas en las rupturas de pareja, y la responsabilidad de los/as progenitores para preservar el bienestar físico y emocional de los mismos ante dichas situaciones.

Antes de adentrarnos en el meollo de la cuestión, es imprescindible que entendamos varios conceptos que están estrechamente relacionados con la temática. En primer lugar, el término parentalidad. Es fundamental distinguir entre ser padres en sentido biológico, o parentalidad biológica, que es la capacidad de procrear o dar vida a un ser humano, y ser padres o madres competentes o parentalidad social, entendida como la capacidad que tiene un padre o una madre de cuidar, proteger y educar a sus hijos, asegurándoles un desarrollo suficientemente 
sano. Evidentemente, ser padre o madre no sólo implica serlo a nivel biológico, sino que conlleva adquirir una serie de responsabilidades para garantizar el bienestar del/a menor. A nivel legal, dicha responsabilidad aparece contemplada en el artículo 154 del Código Civil, aludiendo a otros términos, como Patria Potestad y Guarda y Custodia. El primero de ellos, hace referencia al conjunto de derechos que la ley confiere a los/las progenitores/as, sobre las personas y bienes de sus hijos no emancipados, así como el conjunto de deberes que también deben cumplir los/as padres/madres respecto de sus hijos/as (obligación de estar con ellos, cuidarlos, protegerlos, educarlos, procurarles una formación integral, representarlos legalmente y administrar bienes). La Patria Potestad es un deber al que no se puede renunciar, y cuya negación puede llegar a constituir delito. Sin embargo, es importante discernir entre la titularidad de la Patria Potestad y el ejercicio efectivo de la misma, lo que se refiere a Guarda y Custodia. Ésta última es entendida como vivir, cuidar y asistir a los/as hijos/as en todas las situaciones vitales.

A tenor de la revisión conceptual, nos surge la siguiente pregunta ¿Tienen siempre los/as padres/madres el derecho a ver a los/as menores, independientemente de la situación familiar? Cuando se establece un régimen de visitas, o se habla de niños y niñas en Derecho de Familia, siempre hemos de aludir a la premisa "el mejor interés del menor". Por consiguiente, todos aquellos profesionales inmersos en este proceso, así como los/as progenitores/as, han de velar por los derechos de los niños y las niñas. Es por ello, que los/as padres/madres no tienen siempre, el derecho a ver a sus hijos/as. En este punto, éstos últimos han de reconocer su responsabilidad y poner conciencia en el establecimiento y fortalecimiento del vínculo paterno/materno-filial.

A partir de aquí, nos vamos a centrar en las acciones que se pueden realizar para contribuir a que los/as menores se adapten lo mejor posible a una situación de separación, teniendo en cuenta que esta adaptación está directamente relacionada con la gestión que se realice, en el proceso de ruptura por parte de sus progenitores.

\section{La ruptura familiar como fuente de pérdida y estrés}

Las rupturas de parejas con hijos/as, siempre son experiencias difíciles, las cuales provocan desajustes en todos los miembros del grupo familiar, principalmente en los/as menores. No obstante, para los/as progenitores/as supone la pérdida de compañía y relaciones íntimas, del rol de pareja, de una red social, así como implicaciones económicas.

En relación a los/as hijos/as, la separación de sus padres acarrea, el debilitamiento o la pérdida de la relación con alguno de ellos, así como la pérdida de recursos personales y socioeconómicos. Todo ello conlleva en los niños y niñas una serie de respuestas emocionales, en función de la etapa evolutiva en la que se encuentren. En la etapa preescolar, suelen presentar malestar profundo, un elevado nivel de ansiedad ante la separación, miedo a que los padres los abandonen, un alto índice de regresiones (retroceso en el control de esfínteres, o en la gestión de las emociones, así como en otros logros alcanzados propios del desarrollo psicomadurativo y evolutivo del niño/a) y una escasa capacidad para entender el divorcio y, por ende, una tendencia a autoinculparse por la separación. Los/as menores en edad escolar, manifiestan un nivel moderado de depresión, se preocupan por la salida del hogar de uno de los progenitores y añoran su regreso. Perciben el divorcio como un rechazo

№ 7, 2019. Página | 168 
hacia ellos mismos, y temen, en gran medida, verse desplazados. Durante la pre adolescencia se produce una expresión de sentimientos de cólera y tendencia a culpar a uno de los/as progenitores/as, pudiendo desarrollar sintomatología de carácter somático (dolor de estómago, náuseas, etc.). Y finalmente, en la adolescencia, a pesar de que se sienten apenados y con cierto nivel de ansiedad, en general afrontan el divorcio de manera más sana. El mayor desarrollo cognitivo y emocional, así como la posibilidad de contar con el apoyo de sus iguales y otros adultos de ambientes extrafamiliares, amortiguan los efectos de la separación y facilitan el ajuste a la nueva situación.

Todas estas reacciones son comunes y normales en los/as menores que afrontan el estrés de la separación, y suelen desaparecer aproximadamente en dos años, tras la ruptura. Sin embargo, cuando existen circunstancias que agravan o hacen que la separación se convierta en un foco de conflicto, como, por ejemplo, la violencia de género, a estas reacciones se le suman las consecuencias de la violencia intrafamiliar que vienen sufriendo tiempo atrás. Es en estos casos, donde la evolución y recuperación de los/as menores dependerá fundamentalmente del nivel de conflictividad de los padres y madres en su gestión del proceso de ruptura. Y es por ello, que el tipo de interacción entre los/as progenitores/as determina de manera directa el estrés sufrido por los niños y las niñas, convirtiéndose en el factor que mejor predice cómo se van a adaptar a esta situación a lo largo del tiempo.

\section{Comportamientos desajustados para/con los/as menores en el proceso post-ruptura}

Es fundamental partir de la premisa y de la idea que es responsabilidad de los padres y las madres anteponer siempre, en todo momento, los intereses de los hijos e hijas, muy por encima de los suyos propios. Si no es así se producen situaciones que suponen una amenaza para el desarrollo armonioso y saludable de los críos/as.

Podemos encontrarnos tantos comportamientos como personas y parejas haya, sin embargo, Fernández y Godoy (2002), en sus intentos por estudiar y clasificar dichas conductas, han desarrollado una lista de las situaciones más comunes, que ponen en riesgo el bienestar de los/as menores. Las circunstancias que dan relieve dichos autores son las siguientes:

- Delegar en los hijos/as responsabilidades adultas en la gestión de la ruptura familiar, por ejemplo, la decisión de relacionarse o no con el otro progenitor/a.

- Compartir con los hijos/as sentimientos, emociones, pensamientos o situaciones derivados del proceso de ruptura, así como información escabrosa sobre los motivos de la separación, con el fin de que se posicionen en un sentido u otro.

- Compartir con ellos/as información sobre el procedimiento legal (leerles demandas o denuncias interpuestas, acudir con ellos a citas con los/as letrados/as) con el objetivo de influir en sus preferencias parentales.

- Utilizar a los hijos e hijas para saber qué hace la otra parte (si trabaja o no, si rehacen sus vidas, sobre su vida social...)

- Manifestar conductas y actitudes que conducen a la anulación de la otra figura. Se ignoran, por ejemplo, días especiales, como cumpleaños de alguno de los progenitores, el día de la madre, del padre, eventos por parte de la otra familia... 
Además de ello, son muchas las ocasiones en las que este tipo de comportamiento se extiende al resto de la familia extensa (tíos/as, abuelos/as primos/as, etc.).

- Deslegitimizar la figura de autoridad de alguno de los/as progenitores/as, dejando sin efecto y cuestionando las sanciones, castigos, premios o, en definitiva, el modelo educativo llevado a cabo sobre los hijos/as.

- Utilizar a los hijos e hijas como mensajeros/as para trasladar información de una parte a otra.

- Devaluar la imagen de la ex pareja delante de los hijos/as.

- Depositar y confiarles a los niños/as secretos que no tienen que compartir con la otra parte.

- Trasladarles una valoración de nuestra relación con ellos en términos de sacrificios, como por ejemplo "tengo que trabajar más duro para que vayáis al mismo colegio", "tengo que renunciar a tener pareja para poder cuidar de vosotros". Esto crea en los/as menores sentimientos de culpa, limitando su naturalidad, espontaneidad y marcando su infancia en cuanto al desarrollo psicoevolutivo.

- Expresar actitudes y conductas que provocan el rechazo a la relación con la otra persona.

- Intentar cambiar la figura materna o paterna por la de la nueva pareja, obligándoles a llamarles "mamá" о "papá".

- Dramatizar en las entregas y recogidas, maximizando las emociones o simulándolas.

Todos estos posibles escenarios, conforman situaciones de riesgo, las cuales, si no se erradican a tiempo o no se trabaja sobre ellas, conllevan consecuencias emocionales y conductuales en los hijos e hijas, siendo estas manifestaciones fieles reflejos del contexto que están vivenciando. Fernández y Godoy describieron las consecuencias psicomadurativas y emocionales que una separación conflictiva podía tener en los hijos/as. Describieron ocho tipos de niños y niñas, en función de la ruptura conyugal que hubieran experimentado. En primer lugar, describieron a los/as niños/as hipermaduros, los cuales tienden a mantenerse serios y responsables, reflejando una madurez aparentemente superior a lo que le correspondería por su edad, todo ello con la finalidad de evitarle más sufrimiento a sus progenitores/as. Además de ellos, los/as niños/as espías son utilizados por sus padres o madres para conocer qué es lo que hace la otra parte. Sus respuestas producen una serie de reacciones emocionales desajustadas en el/la progenitor/a que les interroga, que son fáciles de detectar por los niños/as, situación que les produce un elevado estrés. Los/as niños/as en conflicto de lealtades. En esta ocasión, no quieren desagradar a ninguno de sus padres y viven con el temor a hacer o decir algo que pueda molestar a alguno de ellos o desembocar en un nuevo conflicto, reavivando las situaciones estresantes y sintiéndose culpables por ello. Como intentos para evitar estas situaciones mienten y disimulan, ya que sienten que, si son leales a una de las partes, traiciona a la otra, sintiendo la necesidad implícita de posicionarse con alguno de sus padres. Cuando uno de los progenitores rechaza absolutamente al otro hasta negar su existencia ("no quiero saber nada de él/ella, es como si no existiera"), los/as menores aprenden a ocultarlo en su presencia, dando lugar a niños/as divididos, no mencionándolo u ocultando los regalos, entre otras conductas. Es muy probable que no exista una orden explícita de no hablar sobre la otra parte, pero el/la niño/a identifica las reacciones o gestos de molestia, impaciencia, enfado, desatención o distracción, que transmiten el mensaje de desinterés hacia la otra persona. Los/as niños/as mensajeros son utilizados/as 
para transmitir mensajes poco favorables, exigencias, amenazas, insultos, etc. ("Dile a tu madre que la próxima vez que..."). De este modo, los hijos e hijas comienzan a formar parte activa de un conflicto que no les corresponde, y del cual asumen la mayor parte de la responsabilidad. Los/as niños/as colchón, poseen la función de amortiguar la situación entre los progenitores, siendo el punto donde estos últimos descargan la rabia. Sobre ellos recaen insultos, descalificaciones, desvalorizaciones de uno contra el otro, no delatando a ninguno de ellos, ya que sabe que eso podría empeorar las cosas. De este modo, los/as pequeños/as asimilan todo tipo de información negativa sobre ambas o una de las partes, interiorizándolas en la mayoría de los casos, y no expresándola de ninguna manera, lo que afectará muy probablemente a su desarrollo emocional. En la situación de los/as niños/as confidentes, uno de los progenitores vuelca toda la emocionalidad sobre él o ella, con la finalidad de obtener apoyo y alivio. Ante esta situación se produce un proceso de parentificación, en el que los/as hijos/as adquieren roles que no le corresponden, tanto por su edad como por la situación (por ejemplo, se ven en la tesitura de dar consejos, cuidar al padre o la madre que se apoya en ellos, etc.).Finalmente, los autores hacen referencia a los/as niños/as que son víctimas del sacrificio de su madre/padre. Se les hace sentir que son una carga, con verbalizaciones como "Mira todo lo que hago por ti", "He tenido que renunciar a mi vida por ti" o "Si no tuviera hijos...". Este tipo de conductas y expresiones ejercen una elevada influencia en su autoestima, ya que se sienten los únicos responsables de la situación de su hogar.

\section{Pautas de actuación para facilitar la adaptación de los/as niños/as a la nueva situación familiar}

Los/as hijos e hijas en situaciones de conflictividad durante la separación, siempre son víctimas, y en ningún caso deben de asumir ninguna de las funciones o roles que se han expuesto anteriormente. Es responsabilidad de los/as progenitores/as proporcionarles los cuidados y la protección necesaria ante este y cualquier tipo de contexto, y si es necesario, hacer uso de ayuda especializada.

Sin embargo, no se puede delegar todo el trabajo a actores externos (psicólogos, asesores, letrados, mediadores, etc.), ya que supondría eximir a los padres y las madres de la responsabilidad sobre los/as menores. El papel de ellos/as es fundamental, por lo que se van a exponer pautas básicas positivas de actuación en dichas situaciones, alternativas a todas las explicadas hasta ahora, que favorezcan la adaptación de los/as críos/as al proceso de ruptura. Es primordial evitar presiones y respetar el ritmo y las necesidades de ellos/as para conseguir normalizar la relación y fomentar el vínculo paterno/materno-filial.

- Hablar con los/as hijos e hijas acerca de la ruptura, antes de que sea un hecho legal. Es importante proporcionarle a los/as niños/as explicaciones sobre la situación, pero siempre de manera ajustada y adaptada a su etapa evolutiva, desarrollo cognitivo y emocional, obviando cualquier tipo de información escabrosa y/o perjudicial, que pudiera influenciar en la percepción del/la menor sobre la otra parte.

Algunas indicaciones que se pueden tener en cuenta son (Beyer y Winchester, 2010):

- Nunca responsabilizar a la otra parte de la ruptura.

- Asegurarles que, a pesar de la nueva situación, se seguirá cuidando de ellos/as, pero de forma diferente. 
- Trasmitirle que se sabe que quieren al otro/a progenitor/a y eso está bien y nos agrada.

- Animarles a que pregunten cualquier tipo de información o duda que necesiten saber, temor que tengan ante la nueva situación, ya que eso, facilitará que no realicen interpretaciones erróneas, y les ayudará a elaborar y adaptarse al nuevo ambiente.

- Es cierto que, en ocasiones, los/as niños/as han presenciado de manera directa, episodios de elevada conflictividad, por ejemplo, en casos de violencia de género, por lo que manifiestan reticencias a la hora de relacionarse con el padre. Ante este panorama, es recomendable abordarlo con los/las niños/as, atendiendo a las siguientes pautas:

- Asumir la responsabilidad de la conducta y mostrar el reproche y arrepentimiento sobre la misma.

- Mostrar empatía sobre su madre y sobre ellos, y por cómo debieron sentirse en esa situación.

○ Transmitirle que se entiende el enfado que puedan tener, y solicitarles su perdón, sin presionarlos.

- Comprometerse a elegir una conducta alternativa en otra posible situación de enfado, que se podrá trabajar de manera conjunta.

- Animarle a que expresen sus sentimientos y ponerse en el lugar de los/as pequeños/as, a pesar de que sus palabras hieran.

- Hacerles ver mediante verbalizaciones y afecto congruente con las mismas, que ellos/as no tuvieron ningún tipo de responsabilidad en la forma en la que se comportó el progenitor paterno.

- Preocúpate de cómo se siente. Ponte en su lugar. Ante esa premisa, se trata de respetar y validar sus sentimientos y emociones, así como las formas de afrontamiento que vayan desarrollando ante la nueva situación. Es importante que asimilen y que perciban a sus progenitores como un apoyo, como una base en la que se pueden sustentar, a pesar de lo que puedan sentir en determinados momentos. Es imprescindible evitar juicios de valor sobre sus emociones o conductas, ya que se encuentran en pleno periodo de adaptación. Algunas de las afirmaciones o preguntas de las cuales se puede hacer uso son: “CCómo te sientes?, “Me pregunto cómo se sentirá tu muñeca viviendo en dos casas diferentes?", "Dile a tu osito de peluche que yo estoy aquí por si en algún momento necesita ayuda", "Esto debe de ser muy duro para ti ¿cómo podría yo ayudarte?”.

- Considera la pensión de alimentos como una contribución a la crianza y educación de tus hijos. De nuestra experiencia en asuntos de divorcios, así como la revisión bibliográfica realizada, uno de los temas más controvertidos, es la pensión de alimentos. La finalidad de la misma es atenuar lo máximo posible el desequilibrio o el impacto en la calidad de vida de los/as hijos/as, equiparando, en la medida de lo posible, el nivel de vida en ambas casas. 
- Transmitirles con palabras y actitudes que su otro progenitor/a y su familia extensa son importantes también en su vida. Cuando los/as niños/as deciden contarnos algo de manera espontánea y natural, deriva de la consideración que para ellos/as tienen. En esta vertiente, es importante que los receptores de esa información se muestren asertivos, atendiendo al lenguaje verbal y no verbal. Si cuentan actividades que hacen con la otra parte, es principal transmitirle un feed-back positivo, realizar una escucha activa y no menospreciar ningún aspecto de los que haga referencia. En ocasiones, pueden echar de menos al otro/a progenitor/a, por lo que es fundamental respetar esos sentimientos. Es recomendable ofrecerles la posibilidad de que telefoneen, si así se pueden sentir mejor. Por el contrario, si, transmiten rechazo o crítica hacia la otra parte, es bueno no alimentar esas actitudes, ya que pueden ser el fruto de la convivencia diaria, de frustración de unos deseos inadecuados o de una interpretación errónea de algún hecho en concreto. Pero, si, por otro lado, un padre/madre le está transmitiendo información contraproducente sobre el otro/a, es importante separar la emoción que eso pueda suscitar, y centrar el foco en los/as niños/as y sus propias necesidades, provocando un giro atencional hacia ellos/as.

- Algunas pautas para reducir el estrés del menor a la hora de cumplir con el régimen de visitas establecido, incluyendo pernoctas. Dado que el área familiar del niño/a ha sufrido un fuerte cambio, es considerable que se intenten mantener las demás, en lo referente a sus amigos/as, centro escolar, actividades extraescolares o mascota, la cual se trasladará con ellos, cumpliendo el mismo régimen de visitas. Es importante concederles un periodo de adaptación, en el cual ellos/as marquen el ritmo adecuado, con cada cambio que se lleve a cabo. La actitud y comportamientos que muestren, así como las verbalizaciones que puedan hacer, sirven de guía, ya que serán un fiel reflejo de sus pensamientos, emociones y sentimientos. De cara a las pernoctas, podría resultar útil resaltar aquellos aspectos positivos de pasar tiempo con el progenitor no custodio, proponiéndoles y ofreciéndoles actividades de su gusto.

- Por otro lado, la concepción espacio-temporal de los/as niños/as difiere de la nuestra, atendiendo su desarrollo psicomadurativo y cognitivo. Eso, unido a la nueva situación, y al cambio de domicilio que tiene que llevar a cabo, puede provocar en ellos/as sensación de incontrolabilidad, que dificulte aún más el proceso de adaptación. Es por ello que, sería producente anticipar con ellos y organizar las visitas establecidas, lo que les favorecería tener un mayor control sobre sus vidas y poder prever los cambios y organizarse en función de ellos.

Asimismo, es relevante regular el cumplimiento del tiempo en el que le corresponde a cada una de las partes estar con ellos, ya que, si surge cualquier imprevisto que impida poder recogerlos o un retraso, es interesante comunicárselo con la mayor antelación y explicarles el motivo. Con este tipo de actuaciones podrían evitarse sentimientos de abandono, disminución del autoestima y culpa.

Una vez que concluye el tiempo con ellos/as, transmitirle el afecto que se le tiene, evitando sentimientos negativos (como la desesperanza, tristeza), ya que pueden sentir malestar y provocar conflicto de lealtades. En definitiva, se les ha de transmitir, y ellos/as han de percibir, que no tienen que ocuparse de ninguno de sus progenitores/as.

No 7, 2019. Página | 173 
Con todo ello, se trata de que cada persona y cada familia elabore un plan de actuación propio e individualizado, con el objetivo prioritario de que los/as menores tengan una infancia o adolescencia sana, a pesar de las circunstancias que les han tocado vivir.

\section{Conclusiones}

Hace más de cuatro décadas que se aprobaba en nuestro país la ley del Divorcio, que hoy día rige todos los procesos de familia que llegan a los Juzgados. Sin embargo, desde una perspectiva psicosocial y forense, aún queda mucho camino por recorrer, ya que la cultura del divorcio en España presenta importantes limitaciones y carencias, a la vista de los datos sobre menores expuestos a violencia intrafamiliar, así como la saturación en los Juzgados de Familia o $1^{\circ}$ Instancia. Dada la creciente demanda social en esta vertiente, la labor de los/as psicólogos/as ha ido en aumento, atendiendo a todo tipo de actuaciones (evaluativas, exploratorias, asistenciales, orientativas, mediadoras, etc.), evolucionando y adaptándose a las necesidades actuales. Es justo, esa premisa de la cual ha derivado el presente escrito. Cada vez son más las familias que se encuentran en situación de desamparo por parte de los organismos encargados, debido al auge de separaciones sentimentales con menores, colapsando y mermando las funciones de los mismos. Es por ello que, apostando por nuestra labor y vocación, nos vemos en la "obligación" de prestar nuestros servicios y conocimiento a todas aquellas familias que estén pasando por una situación similar, facilitando, en la medida de lo posible, la restructuración y adaptación al nuevo contexto familiar y, principalmente, atender al mejor beneficio del/a menor. A lo largo de las líneas anteriores, se puede percibir la importancia que para nosotras suscita este tema de total actualidad, por lo que deseamos que nuestra labor tenga el mayor alcance posible. Así que, además de asistir, evaluar, peritar y asesorar a las familias en proceso de separación o divorcio, nos resulta imprescindible la transmisión de información a otros/as profesionales, que desempeñan su labor en dicho ámbito. Desde Psicoclinic S. Coop. And., continuamos formándonos y gestando entre nuestras paredes, futuros proyectos encaminados en líneas futuras.

\section{Referencias}

Beyer, R. y Winchester, K. (2010). Cómo explicar el divorcio a los hijos. Un manual para padres. Madrid: Espasa Libros.

Fernández, E. y Godoy, C. (2002). El niño ante el divorcio. Madrid: Pirámide.

Ramírez, M. (2015). Cuando los padres se separan. Alternativas de custodia para los hijos (Guía práctica). Madrid: Biblioteca Nueva. 